\title{
XPS BINDING ENERGIES OF DEEP CORE LEVELS AND THE AUGER PARAMETER - AN APPLICATION TO SOLID SULFUR COMPOUNDS*
}

\author{
T. Chassé, H. Peisert, P. Streubel, R. Szargan and A. Meisel \\ Fachbereich Chemie, Universität Leipzig \\ Linnéstr. 2, O-7010 Leipzig, Germany \\ (Received January 21, 1993)
}

\begin{abstract}
$\mathrm{X}$-ray photoelectron spectroscopy/X-ray excited Auger electron spectroscopy investigations of solid sulfur compounds are reported. Deep $S$ is core level binding energies were measured in addition to the common $S 2 p$ level and the $K L L$ Auger spectra to derive the Auger parameters. Local and solid state effects on the binding energies were identified using both core levels within an initial state-final state framework. The results are discussed in comparison to recent data on phosphorus compounds. The information from the deep core level is emphasized.
\end{abstract}

PACS numbers: 79.60.-i, 71.20.Fi, 73.20.-r

\section{Introduction}

Energy shifts of core level binding energies measured in X-ray photoelectron spectroscopy (XPS) are widely used to derive chemical state information. Similarly, energy shifts due to changes in chemical bonding are well known for the energies of Auger transitions involving core levels, too. A significant increase in information may be obtained by combining binding energies from XPS and energies from Auger transitions from X-ray excited Auger spectroscopy (XAES) to an Auger parameter $\alpha$, as has been first suggested by Wagner [1], e.g.

$$
\alpha^{\prime}=E_{\mathrm{B}}(2 p)+E\left(K L_{2,3} L_{2,3} ;{ }^{1} D_{2}\right) \text {. }
$$

These Auger parameters have been shown to allow chemical state identification, especially in combination with the two-dimensional chemical state plot. Moreover, shifts of the Auger parameters offer an unique way to determine the related shifts of relaxation energies experimentally. More accurately, this requires the incorporation

*This paper is dedicated to Professor Dr. Julian Auleytner on the occasion of his 70th birthday. 
of a second core level binding energy (BE). In the case of a 3 rd period element we obtain $[2,3]$ :

$$
\Delta R_{\mathrm{S}}(2 p 2 p)=\Delta E\left(K L_{2,3} L_{2,3} ;{ }^{1} D_{2}\right)+2 \Delta E_{\mathrm{B}}(2 p)-\Delta E_{\mathrm{B}}(1 s) .
$$

The static relaxation energy $R_{\mathrm{S}}$ is related to the two-hole final state of Auger emission and is assumed to be twice the dynamical relaxation energy $R_{\mathrm{D}}$ relevant for the photoemission process. These relaxation energies reflect the efficiency of core hole screening and are sensitive to both the local chemical bonding of the photoemitting atom in the solid and the polarizability of the solid state environment. Furthermore, changes of the electrostatic potential at the studied atom as a ground state effect can be investigated [2-5].

Many applications of the Auger parameter approaches limit themselves to measurements of an Auger and one binding energy due to unaccessibility of deep core line BEs or other reasons. This approximation neglects the differences of chemical shifts of different core level binding energies. It is the aim of this paper to present preliminary results on core level and Auger energy shifts obtained on solid sulfur compounds which emphasize the additional information from taking into account the $\mathrm{S} 1 s$ core level as has been done earlier for $\mathrm{Si} 1 s[2,4]$ and $\mathrm{P} 1 s[3-5]$.

In Secs. 2 and 3 the experimental details and results are presented and compared to the literature data. Section 4 is devoted to the chemical state information available from the initial state contributions to the BEs. The relaxation energy will be considered in Sec. 5. Finally, the additional information available from measurement of the deep $1 s$ core level BE is emphasized.

\section{Experimental}

The measurements were performed with a VG ESCA 3 photoelectron spectrometer working with a hemispherical analyzer (CIIA) at constant pass energies of 20 or $50 \mathrm{eV}$. The spectrometer had been equipped with a specially prepared Al/Pd mixed anode. Thus, the excitation of S $2 p$ and $\mathrm{S} 2 s\left(\mathrm{Al} K_{\alpha}: h \nu=1486.6 \mathrm{eV}\right)$, $\mathrm{S} 1 s$ core levels ( $\left.\mathrm{Pd} L_{\alpha_{1}}: 2838.6 \mathrm{eV}[6]\right)$, and $\mathrm{S} K L L$ Auger spectra ( $\mathrm{Pd} L_{\alpha}$ and bremsstrahlung) was possible. The X-ray tube was operated at $12 \mathrm{kV}$ and 20 $\mathrm{mA}$. The energy scale was routinely calibrated to reproduce the BEs of $\mathrm{Cu} 2 p_{3 / 2}$ $(932.65 \mathrm{eV})$ and $\mathrm{Au} 4 f_{7 / 2}(84.00 \mathrm{eV})$ as suggested in [7]. To ensure an appropriate alignment at higher energies near the $\mathrm{S} K L L$ the position of the Au $M_{4} N_{7} N_{7}$ Auger peak at $2101.2 \mathrm{eV}$ [8] was checked, too. A further confirmation for linearity and proper alignment of the energy scale is the agreement of the differences of $\mathrm{Na} 1 s$ kinetic energies to the differences of the respective excitation energies. All these energy differences were reproduced within $0.1 \mathrm{eV}$.

We measured the spectra of three single crystalline and six powdered samples. $\mathrm{ZnS}$ single crystal was prepared by weak sputtering and the $\mathrm{FeS}_{2}$ single crystal by rinsing in methanol to remove major surface contaminations.

The crystal structure of the powdered samples were checked by X-ray diffraction. They were inserted into the UHV on a double-sided adhesive tape. Residual carbon contaminations were used to remove shifts due to sample charging 
by aligning to $E_{\mathrm{B}}(\mathrm{C} 1 s)=285.0 \mathrm{eV}$. The spectra were recorded at pressure of $10^{-7}-10^{-6} \mathrm{~Pa}$. The accumulated spectra were analyzed using a least-squares peak fitting routine, resulting in an accuracy of $0.2 \mathrm{eV}$ for the present data.

\section{Core level and Auger spectra}

Electron spectra covering the regions of the sulfur core levels and $K L_{2,3} L_{2,3}$ spectra excited by combined $\mathrm{Al} K_{\alpha}, \mathrm{Pd} L_{\alpha_{1}}$, and bremsstrahlung irradiation are shown for $\mathrm{CaSO}_{4}$ in Fig. 1a, b. Despite the low signal to background ratio for $\mathrm{S} 1 s$ core level the statistics of the spectra are sufficient to obtain energies from these spectra, as is demonstrated by the single line spectra in Fig. 2a. The weak feature near the $\mathrm{S} 1 s$ main line is due to $\mathrm{Pd} L_{\alpha_{2}} \mathrm{X}$-ray excitation. The energy difference to the main line of $5.3 \mathrm{eV}$ and the relative intensity of 9:1 is in good accordance with data reported in [9].

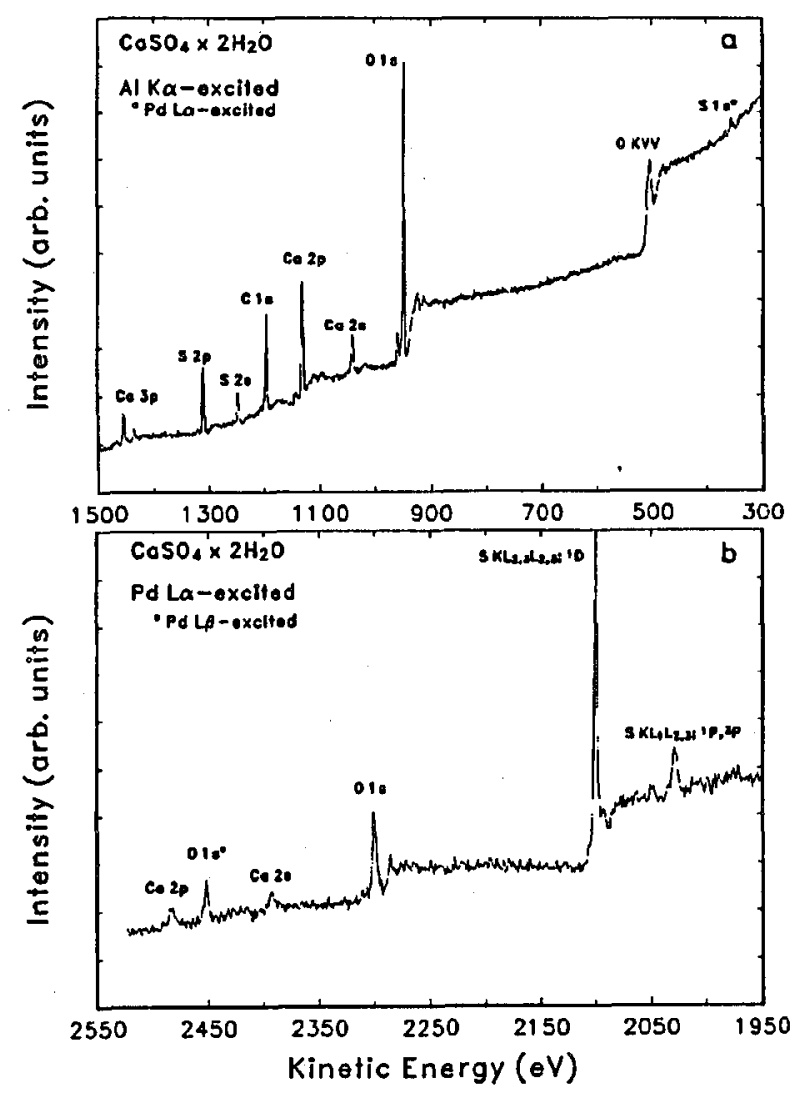

Fig. 1. $\mathrm{Al} K_{\alpha}, \mathrm{Pd} L_{\alpha}$ and bremsstrahlung excited $\mathrm{CaSO}_{4}$ spectra for kinetic energies from 1500 to $300 \mathrm{eV}$ (a) and from 2550 to $1950 \mathrm{eV}$ (b). We used a mixed X-ray source, consisting of the anode materials $\mathrm{Pd}$ and $\mathrm{Al}$. 


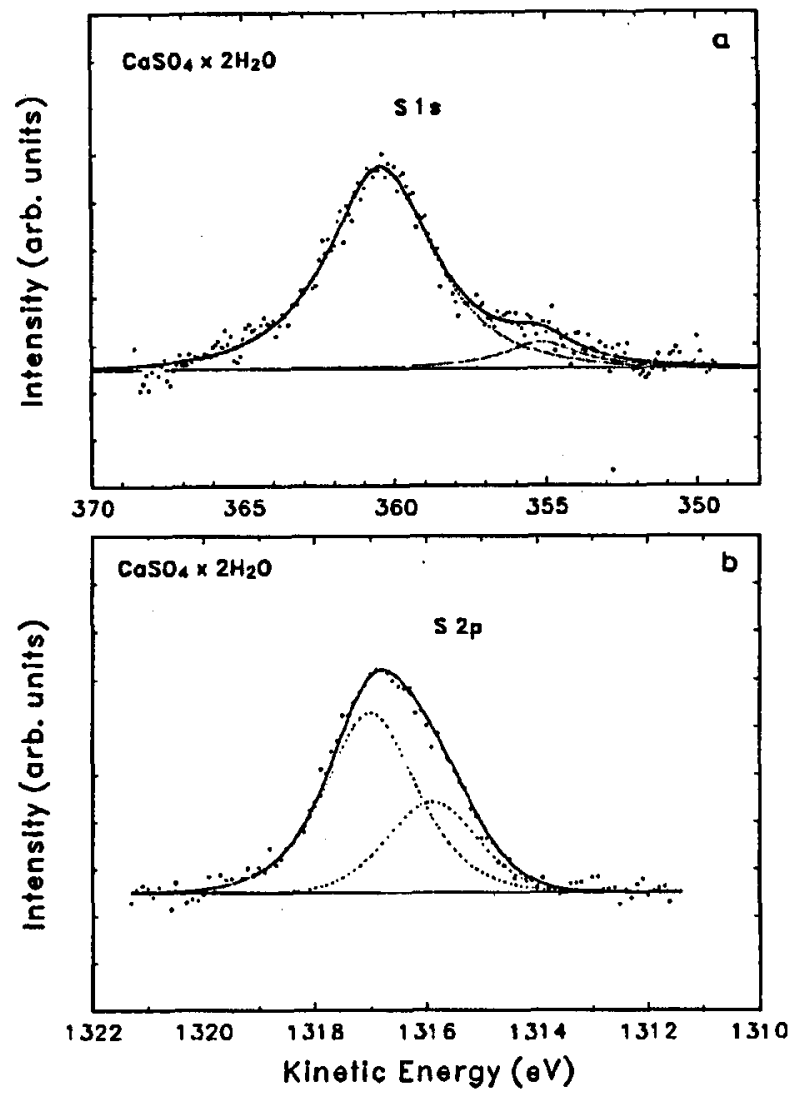

Fig. 2. (a) $\mathrm{Pd} L_{\alpha_{1}}$ and $\mathrm{Pd} L_{\alpha_{2}}$ excited S $1 s$ spectra of $\mathrm{CaSO}_{4}$, (b) $\mathrm{Al} K_{\alpha}$ excited S $2 p$ spectra of $\mathrm{CaSO}_{4}$. The splitting into $2 p_{1 / 2}$ and $2 p_{3 / 2}$ components is indicated.

The $\mathrm{S} 2 p$ core line is displayed in Fig. $2 \mathrm{~b}$. The splitting of this core level into $2 p_{3 / 2}$ and $2 p_{1 / 2}$ components is evident as a weak shoulder only, due to the limited resolution of the unmonochromatized Al $K_{\alpha}$ excitation. Although this splitting can be resolved by the peak fitting routine, we will report energies relevant for the unresolved peak in the following.

The binding energies of the $\mathrm{S} 2 p$ and $\mathrm{S} 1 s$ core levels are given in Table I. Comparison with literature data $[10,12]$ gives rather good agreement considering the difference of the $\mathrm{C} 1 s$ reference energy of $283.7 \mathrm{eV}$ used by Yu et al. [10] and the value of $285.0 \mathrm{eV}$ in this work. There are only a few $\mathrm{S} 1 s$ binding energies known from previous work, and these energies are in reasonable agreement to our data. On the other hand, significantly higher $K L_{2,3} L_{2,3}$ Auger energies $(\approx 4 \mathrm{eV})$ have been reported by Yu et al. [10]. Even after correction for the different reference energies there remains a gap of 2-3 eV in the experimental data of $\mathrm{Yu}$ and in this work as shown in Table II. As described above, we took care in calibrating the energy scale of our instrument to avoid nonlinearities. Unfortunately, there is a 
TABLE I

Core level binding energies and Auger energies in solid sulfur compounds. The energies are given in $\mathrm{eV}$.

\begin{tabular}{|c|c|c|c|c|c|c|}
\hline \multirow[b]{2}{*}{ Compound } & \multicolumn{2}{|c|}{ S $2 p$} & \multicolumn{2}{|c|}{$\mathrm{S} K L_{2,3} L_{2,3} ;{ }^{1} D_{2}$} & \multicolumn{2}{|c|}{$\mathrm{S} 1 \mathrm{~s}$} \\
\hline & $\begin{array}{l}\text { this } \\
\text { work }\end{array}$ & $\begin{array}{l}\text { other } \\
\text { authors }\end{array}$ & $\begin{array}{l}\text { this } \\
\text { work }\end{array}$ & $\begin{array}{l}\text { other } \\
\text { authors }\end{array}$ & $\begin{array}{l}\text { this } \\
\text { work }\end{array}$ & $\begin{array}{c}\text { other } \\
\text { authors }\end{array}$ \\
\hline $\mathrm{ZnS}$ & 162.3 & $\begin{array}{l}161.7^{a} \\
160.15^{b}\end{array}$ & 2113.5 & $\begin{array}{l}2114.4^{a} \\
2118.25^{b}\end{array}$ & 2469.2 & $2469.4^{a}$ \\
\hline $\mathrm{FeS}_{2}$ & 163.0 & - & 2115.8 & - & 2470.2 & - \\
\hline $\mathrm{Na}_{2} \mathrm{~S}_{2} \mathrm{O}_{3} \cdot 5 \mathrm{H}_{2} \mathrm{O}(-2)$ & 162.8 & $160.55^{b}$ & 2111.8 & $2116.35^{b}$ & 2469.8 & - \\
\hline $\mathrm{Na}_{2} \mathrm{~S}_{2} \mathrm{O}_{3} \cdot 5 \mathrm{H}_{2} \mathrm{O}(+6)$ & 168.7 & $166.50^{b}$ & 2107.3 & $2111.70^{b}$ & 2476.5 & - \\
\hline $\mathrm{Na}_{2} \mathrm{~S}_{2} \mathrm{O}_{3}(-2)$ & 162.7 & $161.8^{a}$ & 2112.2 & $2112.3^{a}$ & 2469.5 & $2469.8^{a}$ \\
\hline $\mathrm{Na}_{2} \mathrm{~S}_{2} \mathrm{O}_{3}(+6)$ & 168.6 & $167.8^{a}$ & 2107.6 & $2107.9^{a}$ & 2476.0 & $2477.0^{a}$ \\
\hline $\mathrm{Na}_{2} \mathrm{SO}_{3}$ & 167.6 & $\begin{array}{l}167.9^{a} \\
165.50^{b}\end{array}$ & 2107.3 & $\begin{array}{l}2107.6^{a} \\
2111.60^{b}\end{array}$ & 2475.3 & $2475.9^{a}$ \\
\hline $\mathrm{Na}_{2} \mathrm{SO}_{4}$ & 169.4 & $\begin{array}{l}169.1^{a} \\
167.65^{b}\end{array}$ & 2105.9 & $\begin{array}{l}2105.9^{a} \\
2109.65^{b}\end{array}$ & 2477.7 & $2478.2^{a}$ \\
\hline $\mathrm{CaSO}_{4} \cdot 2 \mathrm{H}_{2} \mathrm{O}^{S}$ & 169.7 & - & 2106.1 & - & 2478.4 & - \\
\hline $\mathrm{CaSO}_{4} \cdot 2 \mathrm{IH}_{2} \mathrm{O}^{P}$ & 169.7 & $167.00^{b}$ & 2105.8 & $2110.80^{b}$ & 2478.4 & - \\
\hline $\mathrm{CaSO}_{4}$ & 169.8 & - & 2105.7 & - & 2478.2 & - \\
\hline
\end{tabular}

${ }^{S}$ single crystal, ${ }^{P}$ powder.

a experimental data from [12], ${ }^{b}$ experimental data from [10].

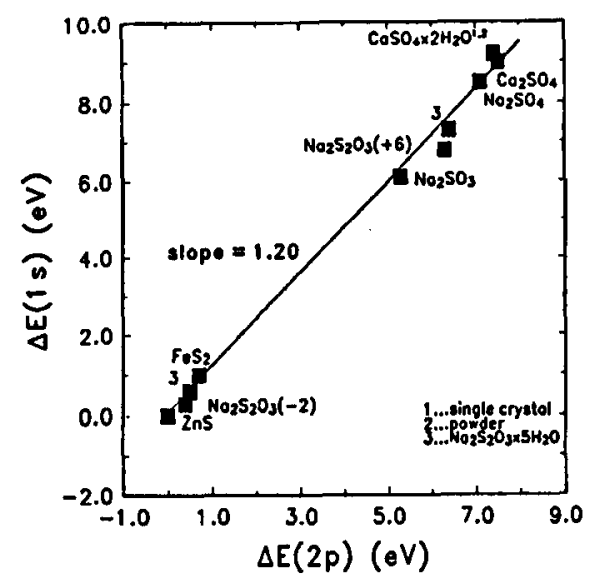

Fig. 3. Correlation of $\mathrm{S} 1 s$ and S $2 p$ binding energies for different sulfur compounds. 
lack of information on that subject in the paper of Yu et al. [10]. Thus, the origin of that rather significant kinetic energy difference is not clear until now.

The binding energy shifts of different core levels are strongly correlated as is demonstrated in Fig. 3 for $\mathrm{S} 1 s$ and $\mathrm{S} 2 p$. An average slope of $\approx 1.20$ of $\Delta E_{\mathrm{B}}(1 s)$ compared to $\Delta E_{\mathrm{B}}(2 p)$ may characterize this correlation reasonably. This value is quite close to 1.20 and 1.16 reported for solid silicon and phosphorus compounds [2-4], respectively. It agrees fairly well with an average slope of 1.16 obtained by Sodhi and Cavell [11] for gaseous sulfur compounds.

TABLE II

Initial and final state effects in solid sulfur compounds. The energies are given in $\mathrm{eV}$.

\begin{tabular}{|c|c|c|c|c|c|}
\hline Compound & $\alpha^{\prime}$ & $\Delta \alpha^{\prime}$ & $\Delta R_{\mathrm{S}}$ & $\Delta V$ & $\Delta \Delta E_{\mathrm{B}}$ \\
\hline $\mathrm{ZnS}$ & 2275.8 & 0 & 0 & 0 & 0 \\
\hline [12] & 2276.1 & 0 & 0 & 0 & 0 \\
\hline [10] & 2278.5 & 0 & - & - & - \\
\hline $\mathrm{FeS}_{2}$ & 2278.8 & 3.0 & 2.7 & 2.05 & 0.3 \\
\hline $\mathrm{Na}_{2} \mathrm{~S}_{2} \mathrm{O}_{3} \cdot 5 \mathrm{H}_{2} \mathrm{O}(-2)$ & 2274.6 & -1.2 & -1.3 & -0.15 & 0.1 \\
\hline$[10]$ & 2276.9 & -1.6 & - & - & - \\
\hline $\mathrm{Na}_{2} \mathrm{~S}_{2} \mathrm{O}_{3} \cdot 5 \mathrm{H}_{2} \mathrm{O}(+6)$ & 2276.0 & 0.2 & -0.7 & 6.05 & 0.9 \\
\hline [10] & 2278.2 & -0.3 & - & - & - \\
\hline $\mathrm{Na}_{2} \mathrm{~S}_{2} \mathrm{O}_{3}(-2)$ & 2274.9 & -0.9 & -0.8 & 0.00 & -0.1 \\
\hline$[12]$ & 2274.1 & -2.0 & -2.3 & -1.05 & 0.3 \\
\hline $\mathrm{Na}_{2} \mathrm{~S}_{2} \mathrm{O}_{3}(+6)$ & 2276.2 & 0.4 & -0.1 & 6.25 & 0.5 \\
\hline$[12]$ & 2275.7 & -0.4 & -1.9 & 5.15 & 1.5 \\
\hline $\mathrm{Na}_{2} \mathrm{SO}_{3}$ & 2274.9 & -0.9 & -1.7 & 4.45 & 0.8 \\
\hline [12] & 2275.5 & -0.6 & -0.9 & 5.75 & 0.3 \\
\hline [10] & 2277.1 & -1.4 & - & - & - \\
\hline $\mathrm{Na}_{2} \mathrm{SO}_{4}$ & 2275.3 & -0.5 & -1.9 & 6.15 & 1.4 \\
\hline [12] & 2275.0 & -1.1 & -2.5 & 6.15 & 1.4 \\
\hline$[10]$ & 2277.3 & -1.2 & - & - & - \\
\hline $\mathrm{CaSO}_{4} \cdot 2 \mathrm{II}_{2} \mathrm{O}^{S}$ & 2275.8 & 0.0 & -1.8 & 6.5 & 1.8 \\
\hline $\mathrm{CaSO}_{4} \cdot 2 \mathrm{H}_{2} \mathrm{O}^{P}$ & 2275.5 & -0.3 & -2.1 & 6.35 & 1.8 \\
\hline$[10]$ & 2277.8 & -0.7 & - & - & - \\
\hline $\mathrm{CaSO}_{4}$ & 2275.5 & -0.3 & -1.8 & 6.6 & 1.5 \\
\hline
\end{tabular}

${ }^{S}$ single crystal, ${ }^{P}$ powder. 


\section{Potential effects}

The potential of the ground state charge distribution affects the core level BEs rather directly. From our set of experimental data we can derive two different measures of this initial state shift contribution. The BE shift difference

$$
\Delta \Delta E_{\mathrm{B}}=\Delta E_{\mathrm{B}}(1 s)-\Delta E_{\mathrm{B}}(2 p)
$$

corresponds to the $K_{\alpha}$ shifts in X-ray emission, which reflects changes of the effective charges of the emitting atoms in different solid state environments. The initial state contribution $\Delta V$ to the core level BE shift mirrors the total effect of both the effective charge and of the environmental charge distribution. It can also be derived from experimental data $[2,5]$. The comparison of the two experimental quantilies $\Delta \Delta E_{\mathrm{B}}$ and $\Delta V$ thus enables us to separate local and environmental potential effects. Some problems concerning the accuracy of that approach have been discussed for phosphorus compounds, recently [5].

In Fig. 4 we observe a correlation of $\Delta V$ and $\Delta \Delta E_{\mathrm{B}}$ (see also Table II). Bolh quantities increase for higher oxidation state of the sulfur atom. This main trend is a well-known feature of chemical shifts in both X-ray emission and photoelectron spectroscopy. Thus, we will not discuss it in detail here.

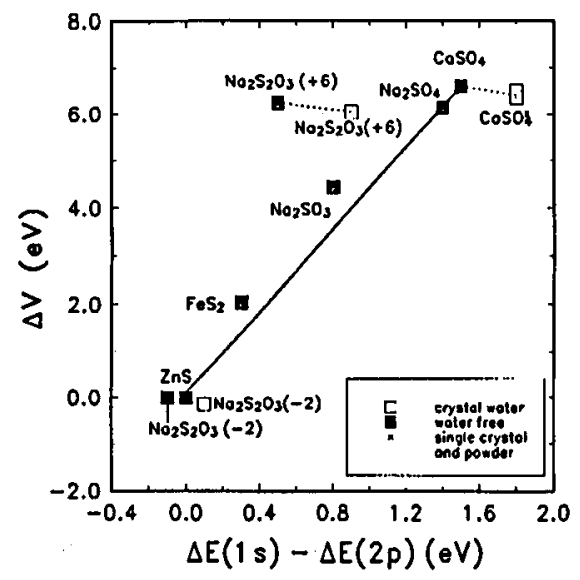

Fig. 4. Comparison of experimentally derived potential effects $\Delta V(2 p)$ of sulfur compounds with differences in binding energy shifts $\Delta \Delta E_{\mathrm{B}}=\Delta E_{\mathrm{B}}(1 s)-\Delta E_{\mathrm{B}}(2 p)$ in reference to $\mathrm{ZnS}$. The lines are to guide the eyes, only.

However, there is a significant increase in $\Delta \Delta E_{\mathrm{B}}$ for all the crystal water containing compounds with respect to the water-free references, indicating additional charge transfer from the sulfur due to the $\mathrm{H}_{2} \mathrm{O}$ molecules in the lattice.

On the other hand, smaller $\Delta \Delta E_{\mathrm{B}}$ for $\mathrm{Na}_{2} \mathrm{~S}_{2} \mathrm{O}_{3}$ than for $\mathrm{Na}_{2} \mathrm{SO}_{4}$ provides direct evidence for reduced charge transfer from the sulfur $(+6)$ atom in the center of the sulfate unit towards the neighbor atoms, as expected from simple electronegativity arguments. The rather high $\Delta V$ measured for the $\mathrm{S}(+6)$ atom in 
thiosulfate may be explained by a reduced environmental potential due to the $S-S$ bond length $(2 \AA)$. The increase in this nearest neighbor distance compared to the $\mathrm{S}-\mathrm{O}$ bond lengths in sulfates $(1.4 \AA)$ is accompanied by such a reduction, which can be easily seen from a Madelung approach of the environmental potential.

\section{Relaxation energy}

In Table II the Auger parameters $\alpha^{\prime}$ and the corresponding shifts $\Delta \alpha^{\prime}$ and $\Delta R_{\mathrm{S}}$ are compared. A chemical state plot is shown in Fig. 5. As usual, core level binding energies (S $2 p)$ and $K L L\left({ }^{1} D_{2}\right)$ Auger energies are compared, directly. Wagner's Auger parameter $\alpha^{\prime}$ is indicated by diagonal lines. We have enclosed further data from Nishikida and Ikeda [12]. There is an evident separation in Fig. 5 between more covalently bonded sulfur compounds at the lower photoelectron BEs and higher Auger energies and polar-covalent compounds on the other side. However, rather similar Auger parameters $\alpha^{\prime}$ are observed for sulfides like $\mathrm{ZnS}$ and sulfates like e.g. $\mathrm{CaSO}_{4}$ despite their different formal oxidation states.
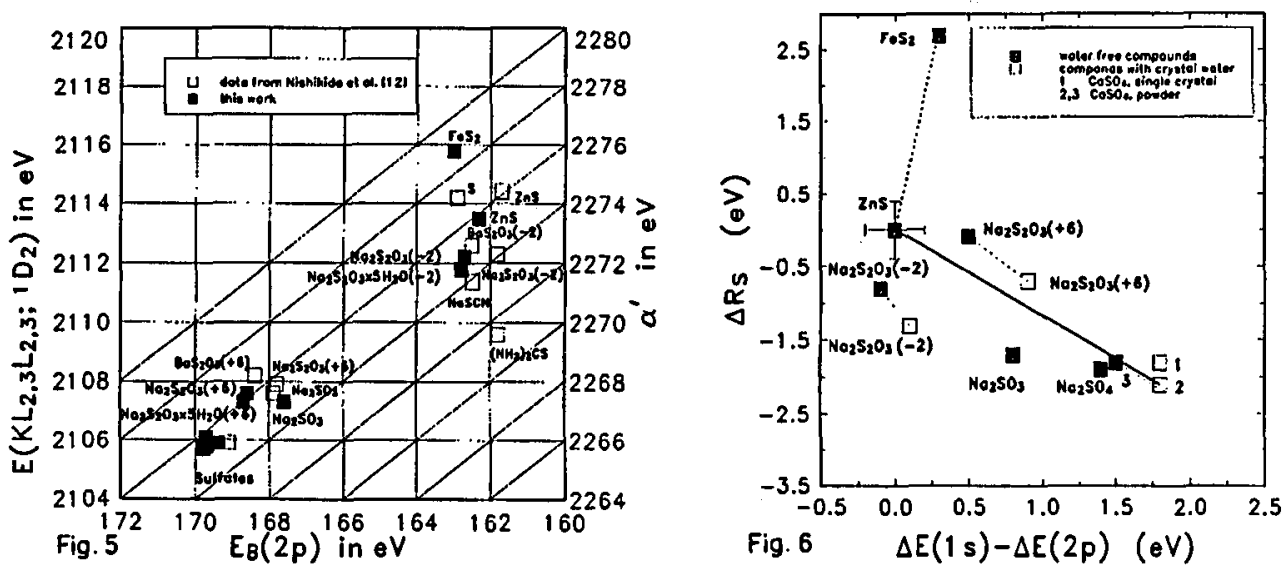

Fig. 5. Two-dimensional chemical state plot for solid sulfur compounds. For comparison with other compounds the reference compound $\mathrm{ZnS}$ was used.

Fig. 6. Experimentally derived shifts of extra-atomic static relaxation energy $\Delta R_{\mathrm{S}}(2 p, 2 p)$ of solid sulfur compounds in comparison with difterences in binding energy shifts $\Delta \Delta E_{\mathrm{B}}$ in reference to $\mathrm{ZnS}$. The lines are to guide the eyes.

Relaxation energies $\Delta R_{\mathrm{S}}$ as derived from our data are compared to $\Delta \Delta E_{\mathrm{B}}$ in Fig. 6. There is no clear trend characterizing all the data. However, looking at the compounds with fourfold coordinated sulfur only, the relaxation energy decreases systematically with increasing $\Delta \Delta E_{\mathrm{B}}$ and thus effective charge on the photoemitting atom. This trend may be related to the reduced valence charge contribution to the core hole screening which is well known from calculations for free ions [13]. The relaxation energy is largest for $\mathrm{FeS}_{2}$ among the compounds investigated. 
The screening of the core hole as reflected by the relaxation energy is compared to the refractive index $n$ of the respective solids in Fig. 7. This macroscopic quantity was arranged to be proportional to the polarization charge $(1-1 / \varepsilon) e$. In this manner it reflects the change in polarization energies for these sulfur compounds. We additionally incorporated experimental relaxation energies as estimated from Nishikida et al. [12] and Yu et al. [10]. In case of the latter authors the relaxation energies have been calculated according to Eq. (2) using the approximation $\Delta E_{\mathrm{B}}(1 s)=1.20 \Delta E_{\mathrm{B}}(2 p)$. The different data sets have been aligned at the data point for $\mathrm{ZnS}$.

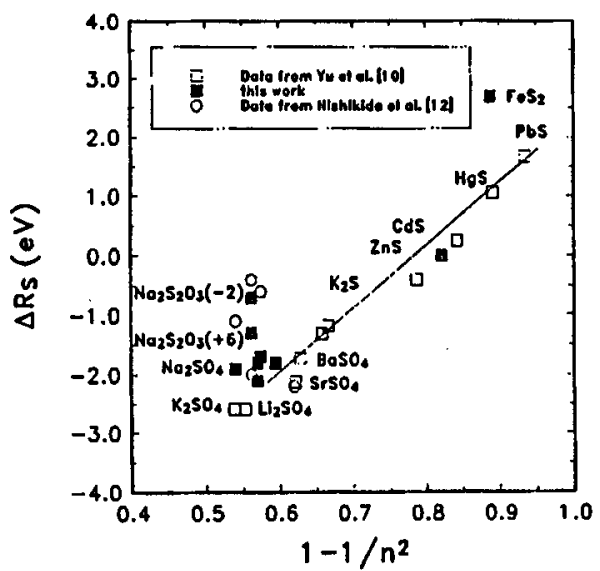

Fig. 7. Experimentally derived shifts of extra-atomic relaxation energies $\Delta R_{S}(2 p, 2 p)$ of solid sulfur compounds versus refractive index $n$. The solid line is to guide the eyes. $\mathrm{ZnS}$ was chosen as reference compound.

There is a clear correlation of the experimental relaxation energies to the refractive index which is indicated by the solid line in the figure (to guide the eye). In this manner, the importance of the polarizability of the whole sample for photohole screening is emphasized. This tendency has been observed for solid phosphorus compounds, recently [5] and even in previous work on other systems. However, the remaining scatter in the present data for sulfur compounds is of considerable size. The dielectric screening of the photoholes by the solid state environment may not be sufficient to explain the experimental differences in the Auger parameters in a simple way.

\section{Concluding remarks}

There are several features worth noting with respect to the use of the binding energy of a deep core level in applications of the Auger parameter concept. First, a much more realistic quantitive approach of relaxation energies may be obtained by using Eq. (2) compared to more common use of Wagner's Auger parameter $\alpha^{\prime}$ (Eq. (1)) only. 
The binding energy of the deep core level reflects the bonding situation similar to that of more shallow levels like $S 2 p$. However, the binding energy shift difference $\Delta \Delta E_{\mathrm{B}}$ of different core levels provides an experimental measure of the local effective charge in the ground state. Although being equivalent in energy to the well-known $K_{\alpha}$ shifts in X-ray emission spectroscopy, $\Delta \Delta E_{\mathrm{B}}$ from XPS is much more surface sensitive than the bulk sensitive $K_{\alpha}$ shifts.

Furthermore, contributions of the solid state environment to the initial state or potential contribution may be separated from local charge effects by comparing $\Delta \Delta E_{\mathrm{B}}$ with $\Delta V$, at least qualitatively. Both quantities are available from application of the Auger parameter concept.

Thus, the impact from the knowledge of deep core level energy shifts $\Delta E_{\mathrm{B}}(1 s)$ to the Auger parameter concept is considerable. This has been shown here for some solid sulfur compounds, and it had been demonstrated in more detail for phosphorus compounds, elsewhere $[4,5]$. It may justify the additional efforts to measure such lines in special cases.

\section{References}

[1] C.D. Wagner, Faraday Discuss. Chem. Soc. 60, 291 (1975).

[2] P. Streubel, R. Fellenberg, A. Reif, J. Electron Spectrosc. Relat. Phenom. 34, 261 (1984).

[3] R. Franke, T. Chassé, P. Streubel, A. Meisel, J. Electron Spectrosc. Relat. Phenom. 56, 381 (1991).

[4] P. Streubel, R. Franke, T. Chassé, R. Fellenberg, R. Szargan, J. Electron Spectrosc. Relat. Phenom. 57, 1 (1991).

[5] T. Chassé, C. Urban, R. Franke, P. Streubel, A. Meisel, J. Electron Spectrosc. Relat. Phenom., submitted.

[6] J.A. Bearden, X-Ray Wavelengths, US Atomic Energy Commission, Oak Ridge 1964.

[7] M.P. Seah, Surf. Interface Anal. 14, 488 (1989).

[8] C.D. Wagner, J. Vac. Sci. Technol. 15, 518 (1978).

[9] M.A. Blochin, Physik der Röntgenstrahlen, VEB Verlag Technik, Berlin 1957.

[10] X.R. Yu, F. Liu, Z.Y. Wang, J. Electron Spectrosc. Relal. Phenom. 50, 159 (1990).

[11] R.S. Sodhi, R.G. Cavell, J. Electron Spectrosc. Relat. Phenom. 41, 1 (1986).

[12] S. Nishikida, S. Ikeda, Bull. Chem. Soc. Jpn. 51, 1996 (1978).

[13] V.I. Nefedow, V.G. Yarzhemsky, A.V. Chuvaev, E.M. Trishkina, J. Electron Spectrosc. Relat. Phenom. 46, 301 (1988). 